Research Article

\title{
Overexpression of MALAT1 Relates to Lung Injury through Sponging miR-425 and Promoting Cell Apoptosis during ARDS
}

\author{
Lu Wang $\mathbb{D}^{1},{ }^{1}$ Jiao Liu, ${ }^{2}$ Wenjie Xie, ${ }^{1}$ Guang Li, ${ }^{1}$ Lan Yao, ${ }^{1}$ Rui Zhang, ${ }^{1}$ and Bin Xu ${ }^{3}$ \\ ${ }^{1}$ Department of Critical Care Medicine, Renmin Hospital of Wuhan University, Wuhan, China \\ ${ }^{2}$ Department of Critical Care Medicine, School of Medicine, North Ruijin Hospital, Shanghai Jiaotong University, \\ Shanghai, China \\ ${ }^{3}$ Department of Oncology, Renmin Hospital of Wuhan Unversity, Wuhan, China
}

Correspondence should be addressed to Lu Wang; wanglu20171010@163.com

Received 31 March 2019; Revised 21 August 2019; Accepted 19 September 2019; Published 1 December 2019

Academic Editor: Alice M. Turner

Copyright (C) $2019 \mathrm{Lu}$ Wang et al. This is an open access article distributed under the Creative Commons Attribution License, which permits unrestricted use, distribution, and reproduction in any medium, provided the original work is properly cited.

\begin{abstract}
Background. Acute respiratory distress syndrome (ARDS) is a severe form of acute lung injury during which severe inflammatory responses induce cell apoptosis, necrosis, and fibrosis. Metastasis-associated lung adenocarcinoma transcript 1 (MALAT1) is a multiple function long noncoding RNA that was found overexpressed during acute lung injury. However, the roles of MALAT1 in ARDS patients are still unknown. Methods. Total RNA was extracted from the plasma, plasma exosome, and peripheral blood mononuclear cells (PBMCs) from 65 ARDS patients and 36 healthy controls. The MALAT1 and six candidate miRNAs levels were detected by qRT-PCR. The interaction between MALAT1 and miR-425 was predicted using a bioinformatics tool and verified by dual luciferase assay. Exosomes from ARDS patients were cultured with A549 and HFL-1 cells to confirm the delivery of miR-425 by exosomes. Cell apoptosis and viability were determined by flow cytometry and MTT assay. Results. We found MALAT1 was significantly increased in the ARDS patients' plasma and PBMCs. The MALAT1 level in PBMCs was negatively correlated with exosomal miR-425 level. MALAT1 interacted with miR-425 and protected phosphatase and tensin homolog (PTEN) expression in A549 and HFL-1 cells. Exosomes from ARDS patients delivered less miR-425 into A549 and HFL-1 cells and induced cell apoptosis via upregulating PTEN. Conclusion. This study identified increased MALAT1 and decreased miR-425 in ARDS patients and unveiled their roles during the pathogenesis of ARDS.
\end{abstract}

\section{Introduction}

Acute respiratory distress syndrome (ARDS) is a severe form of acute lung injury that occurs in critically ill or wounded patients which is characterized by widespread inflammation in the lungs and reduced oxygen uptake $[1,2]$. During ARDS processes, severe inflammatory responses induce cell apoptosis, necrosis, and fibrotic agents releasing, which finally contribute to the pathogenesis of the lungs [3]. Mortality rate for patients with ARDS is very high, and many survivors suffered from complications such as breathing problems $[4,5]$. Prediction of outcome in patients with ARDS is of major importance for appropriate treatment decisions and resource allocation. However, the complex etiology leads to complicated ARDS diagnosis and treatment. Although many protein-based biomarkers have been identified from patients with ARDS, none of them have been translated for ARDS clinical diagnosis [6].

Metastasis-associated lung adenocarcinoma transcript 1 (MALAT1) is a more than $8000 \mathrm{nt}$ long nonprotein coding RNA (lncRNA), which is highly conserved among mammals $[7,8]$. MALAT1 was first identified related to the poor prognosis of patients with non-small-cell lung adenocarcinoma [9]. Subsequently, increasing evidences indicated that MALAT1 is an important multiple function gene expression regulator, which not only contributes to the progression of tumors but also relates to maintaining normal physiological conditions [10], the aging processes [11], and the immune response $[12,13]$. In an LPS-induced acute lung injury rat model, researchers found that MALAT1 knockdown plays protective roles by upregulating miR-146a [14]. However, the roles of MALA1 in ARDS are still unknown. 
Exosomes are small extracellular vesicles derived from endosomal compartment vesicles budding from the plasma membrane [15]. Importantly, exosomes can be produced by almost all types of cells in culture and in various human body fluids including blood, saliva, urine, and breast milk [16]. As an important part of cell-cell communication, exosomes protect molecules from degradation and deliver specific functional proteins and RNAs from supplier cells to receiver cells [17]. Recently, researchers found that exosomes derived from endothelial progenitor cells ameliorate acute lung injury by transferring miR-126 to target endothelial cells [18]. MALAT1, as a nuclear localized lncRNA, has also been found to be degraded into segments, packaged into exosomes, and finally transferred into target cells [19, 20].

Phosphatase and tensin homolog (PTEN) is a tumor suppressor which can modulate the PI3K pathway by catalyzing degradation of PI3K-generated PIP3 [21]. In this manner, PTEN restrains cell proliferation through inhibiting downstream functions of the PI3K-Akt pathway. PTEN is robustly expressed in normal lung fibroblasts, and the downregulation of PTEN is related to aberrant fibroblast proliferation and collagen secretion during LPS-induced acute lung injury [22-24].

In the present study, we examined the MALAT1 and 6 candidate miRNAs levels in plasma, plasma exosome, and peripheral blood mononuclear cells (PBMCs) from 65 ARDS patients and 36 healthy controls. We analyzed the correlation between MALAT1 and miRNAs. Exosomes coculture with lung fibroblasts, and alveolar epithelial cells were employed to analyze the exosome-delivered MALAT1 function.

\section{Materials and Methods}

2.1. Study Population. 65 ARDS patients and 36 healthy volunteers were obtained from Renmin Hospital of Wuhan University hospital. The clinical characters are listed in Table 1. All ARDS subjects met the Berlin diagnostic definition [5]: timing of ARDS was within 1 week of a known clinical insult or new or worsening respiratory symptoms; chest imaging showed bilateral opacities (not fully explained by effusions, lobar/lung collapse, or nodules); respiratory failure was not fully explained by cardiac failure or fluid overload; and ARDS severity was based on $\mathrm{PaO}_{2} / \mathrm{FiO}_{2}$ ratio (including 31 moderate ARDS patients and 34 severe ARDS patients). Patients with diffused alveolar hemorrhage or chronic lung disease were excluded. Treatment with the granulocyte colony-stimulating factor or inhibitors of tumor necrosis factor was also excluded [25]. Patients were enrolled in the study immediately after meeting all inclusion criteria [5]. Institutional review boards of Renmin Hospital of Wuhan University hospital approved this study. Five milliliters of blood samples were collected within the first $24 \mathrm{~h}$. Half of the samples were immediately centrifuged at $3000 \times \mathrm{g}$ for $15 \mathrm{~min}$ at $4^{\circ} \mathrm{C}$. Plasma was removed and stored at $-80^{\circ} \mathrm{C}$ until further assessment. Another half was subjected to cell separation using Ficoll Histopaque (Sigma).

Controls were free of lung, cardiac, infectious, and allergic diseases and without any prescribed chronic diseases.
2.2. Exosome Isolation from Plasma Samples. Exosomes were extracted from plasma using ExoQuick Exosome Precipitation Solution (System Biosciences, Mountain View, Calif). Briefly, plasma was obtained by centrifugation at $3000 \times \mathrm{g}$ for 15 minutes to remove cells and cellular fragments, and subsequent filtration of the supernatant was accomplished through a $0.45-\mu \mathrm{m}$ pore polyvinylidene fluoride filter (Millipore, Billerica, Mass). ExoQuick was added to the supernatants, and exosomes were precipitated by refrigeration at $-20^{\circ} \mathrm{C}$ for 12 hours. Exosome pellets collected by centrifugation at $\times 1500 \mathrm{~g}$ for 30 minutes were dissolved in $20 \mu \mathrm{L}$ PBS.

2.3. RNA Extraction. Total RNA was extracted from cells, plasma, and exosomes using Trizol reagent (Invitrogen, Carlsbad, CA, USA) according to the manufacturer's instructions. cel-miR-39 was added to plasma and EV samples as an external reference. RNA concentration and purity were determined using a model ND-2000 spectrophotometer (Nanodrop Technologies, Wilmington, DE, USA). Only samples with absorbance ratios $260 \mathrm{~nm} / 280 \mathrm{~nm}$ of $\sim 2.0$ and $260 \mathrm{~nm} / 230 \mathrm{~nm}$ of 1.9-2.2 were considered for inclusion in the study.

2.4. Quantitative RT-PCR. Quantitative RT-PCR analysis was used to determine the relative level of selected MALAT1 and miRNAs. Primers for MALAT1 quantification were: F-GAATTGCGTCATTTAAAGCCTAGTT, R-GTTTCAT CCTACCACTCCCAATTAAT; GAPDH: F-ACAGTCAG CCGCATCTTCTT, R-GACAAGCTTC CCGTTCTCAG. The levels of miRNAs were detected by TaqMan miRNA RTReal Time PCR. Single-stranded cDNA was synthesized using TaqMan MicroRNA Reverse Transcription Kit (Applied Biosystems, Foster City, CA, USA) and then subjected to qPCR using miRNA-specific TaqMan MGB probes (Applied Biosystems). Cel-miR-39 was used for normalization in plasma and EV samples. U6 snRNA was used for normalization in cells. Each sample in each group was measured in triplicate, and the experiment was repeated at least three times. The relative MALAT1 and miRNAs levels were compared using the $2-\Delta \Delta \mathrm{Cq}$ method.

2.5. Plasmids Construction. Full length of 8708 bp MALAT1 was cloned into pcDNA3.1 vector between Xho I and Not I restriction sites. To generate MALAT1 luciferase reporter, a segment of $3325 \mathrm{bp}$ of MALAT1, containing predicted miR425 binding site was cloned downstream of the firefly luciferase gene in the pmirGLO plasmid (Promega, Madison, WI, USA). To generate PTEN reporter, a segment of $476 \mathrm{bp}$ segment containing miR-425 binding site was cloned into pmirGLO vector downstream of the firefly luciferase coding region.

2.6. Cell Culture. Human lung fibroblasts HFL-1 were obtained from the American Type Culture Collection (ATCC, Manassas, VA, USA). Human alveolar epithelial A549 cells were purchased from the Chinese National Infrastructure of 
TABLE 1: Clinical characteristics of ARDS patient population.

\begin{tabular}{lccccccc}
\hline Phenotype & $n$ & Age & $\begin{array}{c}\text { Male/ } \\
\text { female }\end{array}$ & Pathogenic factors & Serum IL-1 $\beta(\mathrm{pg} / \mathrm{ml})$ & Lung Ly6G + neutrophil $(\%)$ & $\mathrm{PaO}_{2} / \mathrm{FiO}$ \\
2 & $(\mathrm{~mm} \mathrm{Hg})$ \\
\hline Healthy controls & 36 & 45.3 & $20 / 18$ & NA & $11.1 \pm 6.1$ & $4.1 \pm 0.9$ & $427.8 \pm 49.6$ \\
& 36 & 46.7 & $22 / 14$ & $\begin{array}{c}\text { Primary } \\
\text { pneumonia }\end{array}$ & $50.6 \pm 21.9$ & $11.1 \pm 2.4$ & $96.6 \pm 42.8$ \\
ARDS & 22 & 42.3 & $16 / 6$ & Trauma & $43.6 \pm 17.6$ & $13.5 \pm 2.8$ & $150.2 \pm 67.6$ \\
& 7 & 44.2 & $4 / 3$ & Blood transfusion & $42.5 \pm 19.8$ & $9.1 \pm 4.7$ & $195.9 \pm 99.1$ \\
\hline
\end{tabular}

ARDS: acute respiratory distress syndrome; $\mathrm{PaO}_{2}$ : partial pressure of arterial oxygen; $\mathrm{FiO}_{2}$ : percentage of inspired oxygen; NA: not applicable. Values represent mean $\pm \mathrm{SD}$.

Cell line Resources. All these cells were cultured in Dulbecco's Modified Eagle Medium containing $10 \%$ fetal bovine serum (Hyclone, Logan, UT, USA), $100 \mathrm{IU} / \mathrm{ml}$ penicillin, and $100 \mathrm{IU} / \mathrm{ml}$ streptomycin. All cells were maintained at $37^{\circ} \mathrm{C}$ under an atmosphere of $5 \% \mathrm{CO}_{2}$.

2.7. Dual Luciferase Assay. miR-425 mimic was purchased from GenePharma Co., Ltd. (Shanghai, China). For the luciferase reporter assay, HFL-1 and A549 cells were seeded in 48 -well plates. Luciferase reporter vectors were cotransfected with miR-425 mimic using lipofectamine 2000 (Invitrogen). 48 hours after transfection, cells were lysed, and the cell lysates were subjected to dual luciferase assay with the Dual-Luciferase Assay kit (Promega, Madison, WI, USA). Each treatment was performed in triplicate in three independent experiments. The results are expressed as relative luciferase activity (firefly luciferase/renilla luciferase).

2.8. Immunoblotting. Proteins were extracted from cell samples and then separated by electrophoresis after boiling in sodium dodecyl sulfate/ $\beta$-mercaptoethanol sample buffer. The proteins in the gels were blotted onto a polyvinylidene fluoride membrane (Amersham Pharmacia Biotech, St. Albans, Herts, UK) by electrophoretic transfer. After blocking by $5 \%$ nonfat milk for 1 hour at room temperature, the membrane was incubated with rabbit anti-PTEN polyclonal antibody (Abcam, Cambridge, MA, USA) or mouse anti- $\beta$-actin monoclonal antibody (Santa Cruz Biotechnology Inc., Santa Cruz, CA, USA) overnight at $4^{\circ} \mathrm{C}$. After washing three times, the membranes were incubated with horseradish peroxidase-conjugated goat anti-rabbit or rabbit anti-mouse secondary antibody for another 2 hours at room temperature. Detection by the chemiluminescence reaction was carried out using ECL kit (Pierce, Appleton, WI, USA). The $\beta$-actin signal was used as a loading control.

2.9. Cell Proliferation Assay. Cell proliferation was estimated by the 3-(4,5-dimethylthiazol-2-yl)-2,5-diphenyltetrazolium bromide (MTT) assay. A549 and HFL-1 cells were seeded in wells of 96-well plates at low density $\left(2 \times 10^{3}\right)$ in DMEM medium and allowed to attach overnight. The cells were then cocultured with exosomes from ARDS patients or healthy controls. Twenty microliters of MTT $(5 \mathrm{mg} / \mathrm{mL}$ ) (SigmaAldrich) were added to each well $48 \mathrm{~h}$ after coculture, and the cells were incubated for further $4 \mathrm{~h}$. The absorbance was recorded at $570 \mathrm{~nm}$ with a 96 -well plate reader after addition of dimethyl sulfoxide (DMSO).

2.10. Flow Cytometry. A549 and HFL-1 cells were seeded in wells of 12 -well plates at low density $\left(3 \times 10^{5}\right)$ in DMEM medium and allowed to attach overnight. The cells were cocultured with exosomes from ARDS patients or healthy controls for 48 hours and then subjected to flow cytometry after staining by FITC-Annexin $\mathrm{V}$ and propidium iodide. We followed the methods of $\mathrm{Li}$ et al. [26].

2.11. Statistical Analysis. Data were analyzed using SPSS Statistical Package version 19.0 (SPSS Inc., Chicago, IL, USA). Two-tailed Student's $t$-test was used to calculate statistical significance between two comparator groups. The correlation analysis was analyzed by $\chi^{2}$-analysis. A twotailed $P$ value $<0.05$ was considered significant.

\section{Results}

To understand the roles of MALAT1 in ARDS, the MALAT1 level in plasma, PBMCs, and plasma exosomes was quantified by RT-PCR. We found the MALAT1 level was significantly increased in the plasma and PBMCs from ARDS patients (Figure 1(a)). It is known that some miRNAs were disturbed when ARDS occur; thus, we detected 6 candidate miRNAs in the exosome (Figure 1(b) and Figure S1). We chose these miRNAs because these miRNAs' level altered in the ARDS patients or mouse models [27-31]. We found miR-425 and miR-125b were significantly reduced in the patients with ARDS (Figure 1(b)). It is reported that MALAT1 can function as a miRNA sponge, protecting gene expression through interaction with miRNAs $[32,33]$. So, we hypothesize that MALAT1 level in the cells may relate to the miRNAs level in the extracellular fluids. After correlation analysis, we found MALAT1 level in the PBMCs was negatively correlated with plasma miR-425 in ARDS patients and healthy controls (Figure 1(c)).

Subsequently, we predicted the interaction between MALAT1 and miR-425 using bioinformatics tool: RNAhybrid ([https://bibiserv2.cebitec.uni]). We found there are at least two regions in the MALAT1 which have the potential to bind with the miR-425 seed sequence (Figure 2(a)). To confirm the interaction between MALAT1 and miR-425, we cloned a segment of $3325 \mathrm{bp}$ of MALAT1 into pmirGLO vector, following firefly luciferase coding region to obtain the 

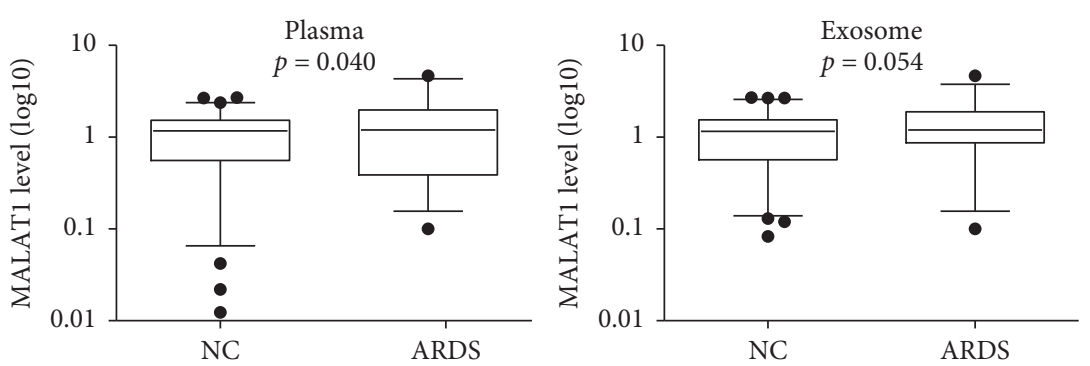

(a)
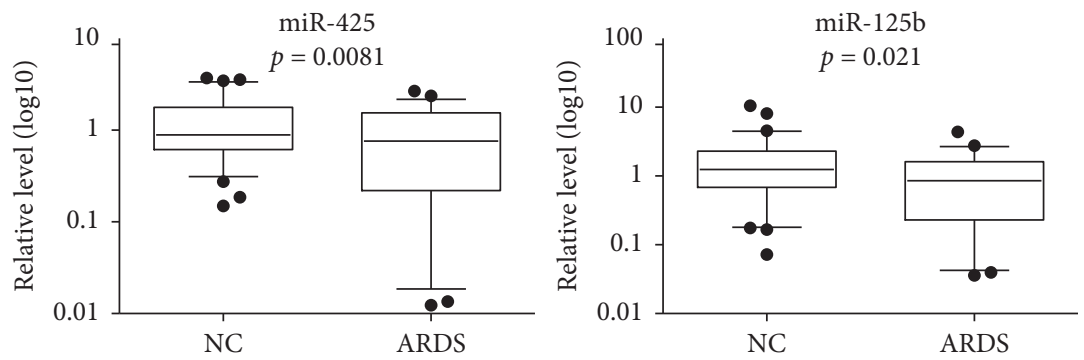

(b)
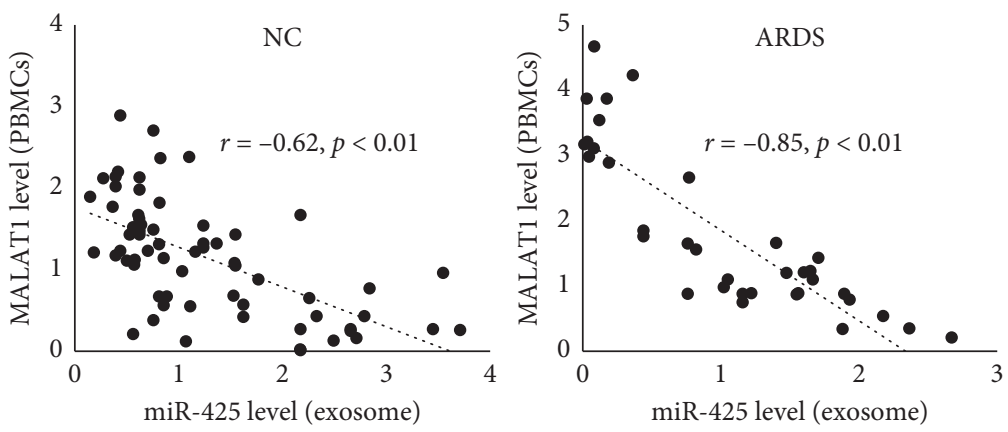

(c)

FIgURE 1: MALAT1 level increased in ARDS patients' plasma and PBMCs, and negatively correlates with miR-425 level in exosomes. (a) Total RNAs were extracted from plasma, plasmal exosomes, and PBMCs from ARDS patients and healthy controls, and the MALAT1 level was quantified by qRT-PCR. (b) Total RNAs were extracted from plasmal exosome and candidate miRNA level was detected by qRTPCR. Results were shown as box-whisker diagram and data out of 95\% CI were shown as dots. (c) Correlation between PBMCs MALAT1 level and exosomal miR-425 level.

reporter vector. HFL-1 and A549 cells were transfected with MALAT1 reporter vector and miR-425 mimic for 48 hours. Cells were lysed for dual luciferase assay. As shown in Figure 2(b), the relative luciferase activities were significantly repressed by miR-425 mimic when compared with control oligo.

To further unveil the correlation between cellular MALAT1 and exosomal miR-425, we constructed wildtype and miR-425 interaction sites deleted mutant MALAT1 expression vector. We overexpressed wildtype or mutant MALAT1 in A549 and HFL-1 cells, and the level of miR-425 in cells and medium was detected by qRT-PCR. As shown in Figure 2(c), the MALAT1 level was successfully overexpressed up to more than twofold in A549 and HFL-1 cells. Meanwhile, the miR-425 levels in the medium were significantly reduced when wildtype MALAT1 was overexpressed in A549 and HFL-1 cells (Figure 2(d)). However, no significant change of miR-425 level in the cells was found in these two cell lines (Figure S2). Meanwhile, when MALAT1 was knocked down by siRNA (Fig. 2E), the miR-425 level in the medium was significantly increased (Fig. 2F). These results indicated that MALAT1 inhibited miR-425 secretion out of the cells but did not repress miR-425 expression in the cells.

To explore the function of the exosomes from ARDS patients, A549 and HFL-1 cells were cocultured with exosomes from ARDS patients and healthy controls for 72 hours. As shown in Figure 3(a), the miR-425 level was significantly decreased in the cells cocultured with ARDS patients' exosomes. Meanwhile, the exosomes from ARDS patients can increase the luciferase activity in the cells transfected with PTEN reporter vector (Figure 3(b)). Immunoblotting was employed to detect the endogenous PTEN protein level in the cells after coculture, and the results showed that PTEN was increased in the cells cocultured with ARDS patients' exosomes (Figure 3(c)). These results indicated that exosomal miR-425 is functional and can be delivered into receiver cells. 
Position 4365-4385

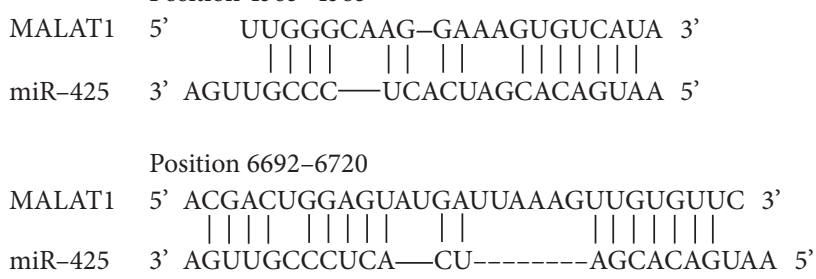

(a)

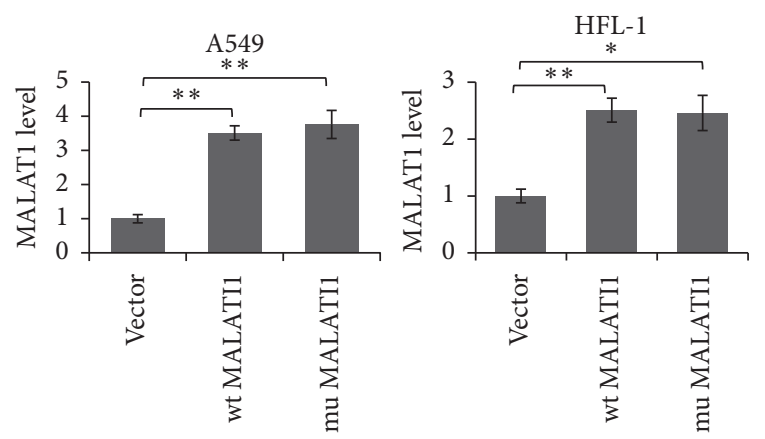

(c)

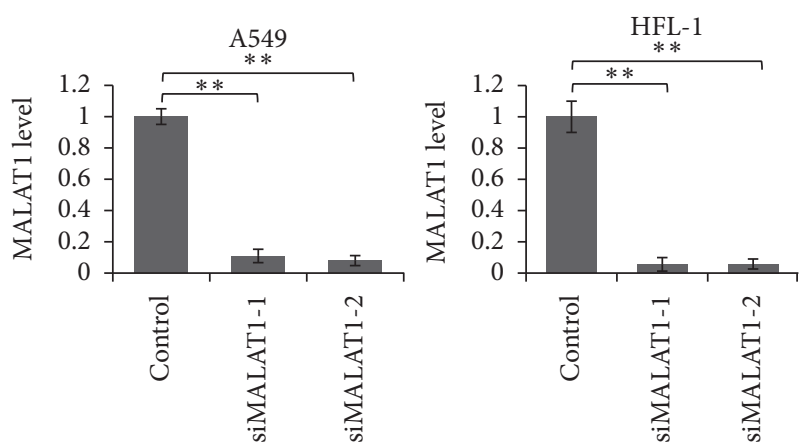

(e)

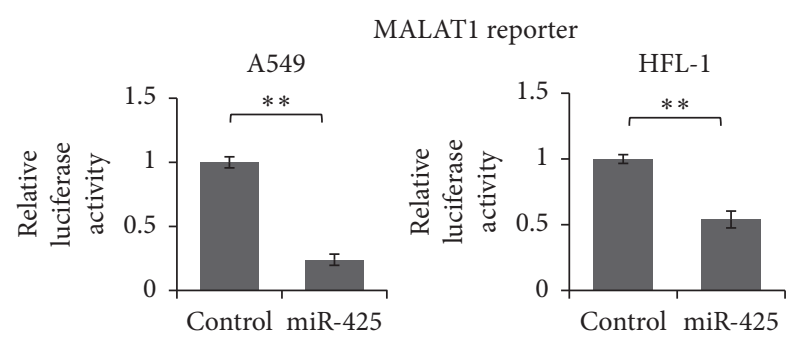

(b)
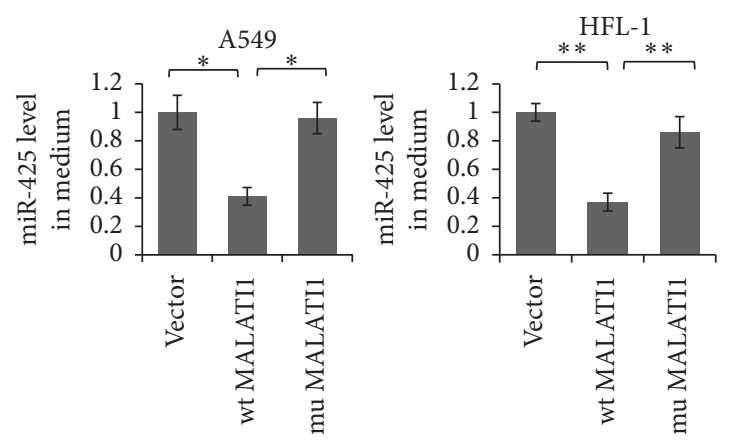

(d)
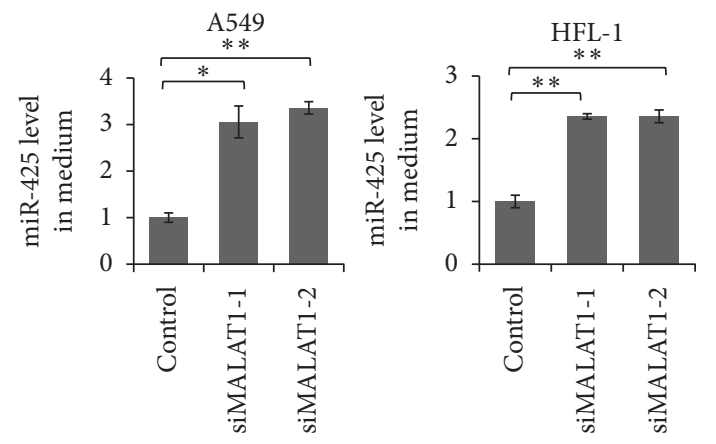

(f)

Figure 2: MALAT1 directly interacting with miR-425 and up-regulated PTEN expression through sponging miR-425. (a) Schematic diagram of predicted interaction between MALAT1 and miR-425. (b) Dual luciferase assay. A549 and HFL-1 cells were transfected with MALAT1 reporter vector and miR-425 mimic or control oligo for 48 hours. Cells were lysed and subjected to luciferase assay. Results were analyzed by Student's $t$-test, and $p<0.05$ was considered significant. (c) A549 and HFL-1 cells were transfected with wildtype or mutant MALAT1 expression vector, with empty vector as control. The MALAT1 level was detected 48 hours after transfection. MiR-425 level in the cell culture medium was detected by qRT-PCR at the same time (d). (e) A549 and HFL-1 cells were transfected with MALAT1 specific siRNAs for 48 hours. The MALAT1 level in A549 and HFL-1 was detected by qRT-PCR. (f) The miR-425 level in the medium was quantified by qRT-PCR. Results were analyzed by $t$-test, and $p<0.05$ was considered significant. ${ }^{*} p<0.05,{ }^{* *} P<0.01$.
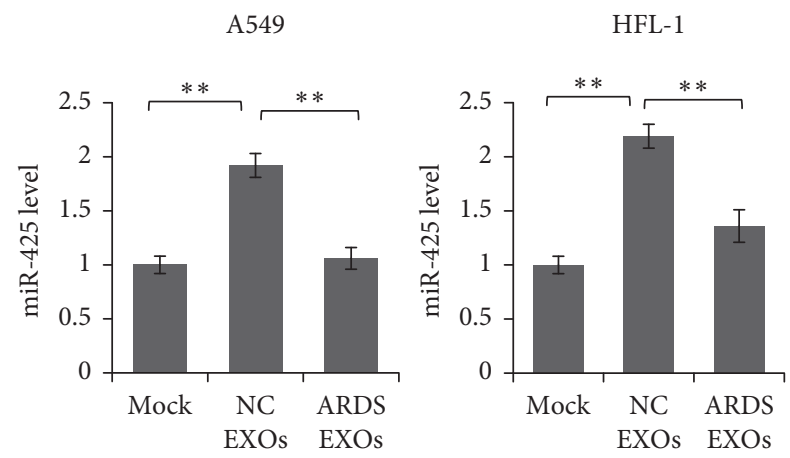

(a)

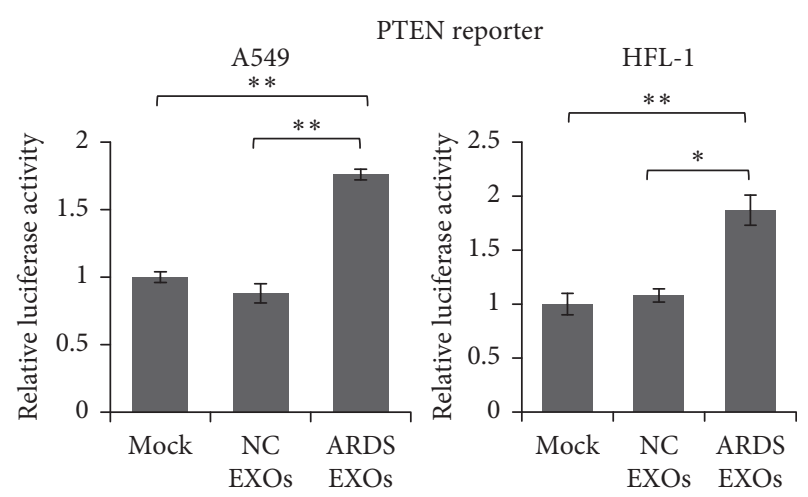

(b)

FIgUre 3: Continued. 

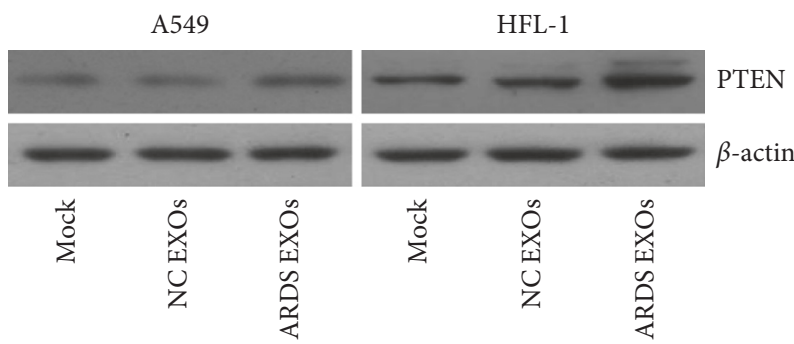

(c)

FIgURE 3: Exosome from ARDS patients' plasma delivered less miR-425 into target cells and did not repress PTEN expression. (a) A549 and HFL-1 cells were cocultured with exosomes from ARDS patients or healthy controls. The miR-425 level was detected by qRT-PCR 48 hours after coculture. (b) A549 and HFL-1 cells were transfected with PTEN reporter and then cocultured with exosomes from ARDS patients or healthy controls. The luciferase activity was detected 48 hours after coculture. Results were analyzed by $t$-test, and $p<0.05$ was considered significant. ${ }^{*} p<0.05,{ }^{* *} P<0.01$. (c) A549 and HFL-1 cells were cocultured with exosomes from ARDS patients or healthy controls. The PTEN protein level was determined by immunoblotting.
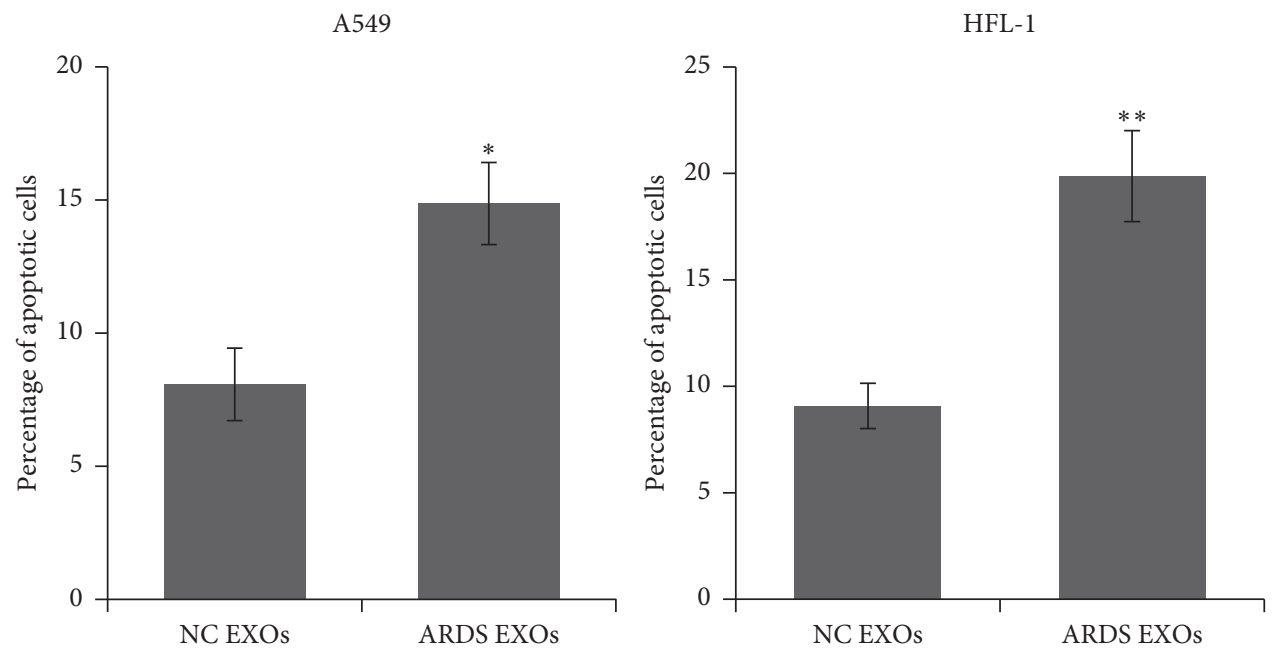

(a)
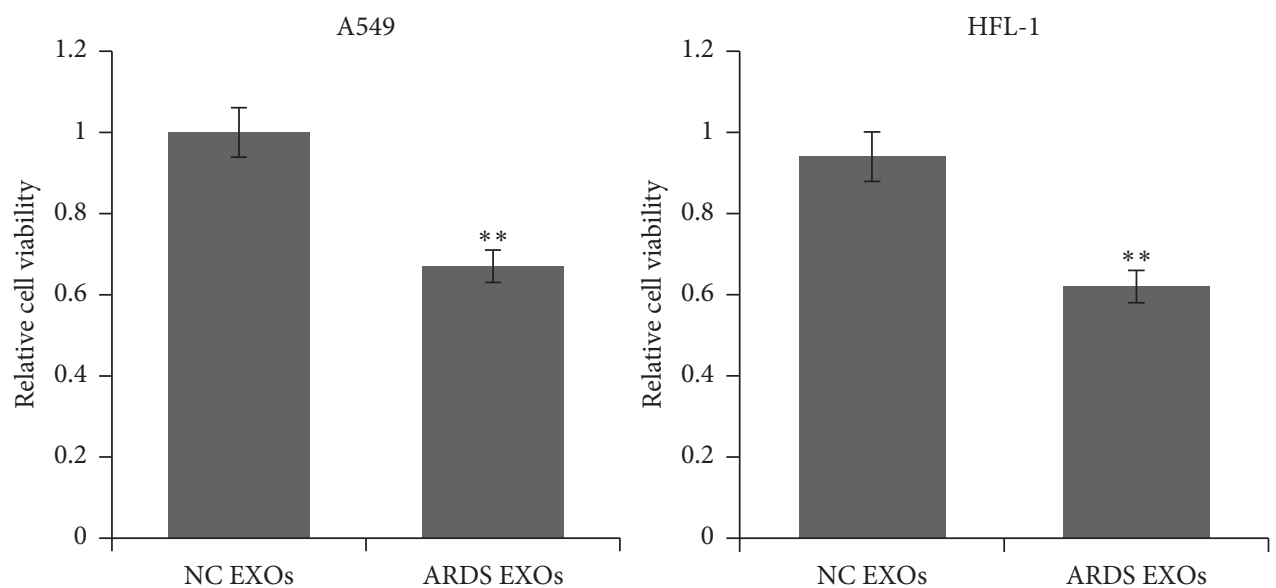

(b)

FIGURE 4: Exosomes from ARDS patients promoted cell apoptosis and inhibit cell proliferation. A549 and HFL-1 cells were cocultured with exosomes from ARDS patients or healthy controls. Apoptotic cells were detected by flow cytometry after FITC-Annexin V and PI staining (a). Cell viability was determined by MTT assay (b). Results were analyzed by Student's $t$-test, and $p<0.05$ was considered significant. ${ }^{*} p<0.05$ and ${ }^{* *} P<0.01$. 
PTEN is an important tumor suppressor and induces cell apoptosis, so we detected the cell apoptosis and viability using A549 and HFL-1 cells after coculturing with exosomes. We observed increased apoptotic cell number (Figure 4(a)) and reduced cell viability (Figure 4(b)) in the cells cocultured with ARDS patients' exosomes.

\section{Discussion}

Acute respiratory distress syndrome (ARDS) is a respiratory failure which is associated with severe inflammatory reactions, interstitial and alveolar edema, cell infiltration into the alveolar space, and endothelial damage [34]. MALAT1 was found upregulated and relates to activation of nuclear factor kappa-light-chain-enhancer of activated B cells (NF- $\kappa \mathrm{B})$ signaling in the lipopolysaccharide (LPS)-induced acute lung injury model [35]. MALAT1 was first identified as a pre-mRNA splicing regulator and mainly located in the cell nucleus [36]. Subsequently, MALAT1 was also found related to DNA double-strand break repair pathway [7]. Although MALAT1 is a nucleus-localized molecule, it has been found to be packaged into exosomes and delivered into the microenvironment and target cells [37, 38]. In this present study, we detected MALAT1 level in the plasma, plasmal exosomes, and PBMC from ARDS patients and want to explore the roles of MALAT1 during the pathogenesis of ARDS. We found the MALAT1 level was extremely high in the PBMCs from ARDS patients and negatively correlates with exosomal miR-425 level.

It is reported that miR-425 can promote cell proliferation in gastric cancer and contribute to the invasion and metastasis in hepatocellular carcinoma by targeting PTEN $[39,40]$. However, the function of miR-425 in ARDS is still unknown. Herein, we confirmed that miR-425 can also repress PTEN expression in A549 and HFL-1 cells. Using dual luciferase assay and immunoblotting, we confirmed that MALAT1 can "sponge" miR-425 in the cells, protect PTEN expression, and inhibit miR-425 secretion into the medium. Thus, these evidences indicated that exosome-delivered miR-425 is an important cell apoptosis inhibitor in the lung microenvironment, and we hypothesized that overexpressed MALAT1 in ARDS patients may promote cell apoptosis and contribute to the lung pathophysiological process by sponging miR-425.

It is reported that PTEN can be packaged into exosomes and delivered into recipient cells [41, 42]. In the exosome coculture experiment, we observed increased miR-425 level in the NC exosome cocultured A549 and HFL-1 cells. However, as the target of miR-425, the PTEN protein level was similar in these two groups which indicated that exosome-delivered PTEN protein may increase the PTEN level in exosome cocultured cells.

Through coculture experiments, we confirmed that ARDS patients' exosomes delivered less miR-425 to the target cells, and induced cell apoptosis by upregulating PTEN expression. These results unveiled the biological role of increased MALAT1 in the cells and decreased miR-425 in the exosomes during ARDS. However, there are still some defects about this study. We found exosomal miR-425 level was reduced, and these exosomes delivered less miR-425, but we still do not know where these exosomes are from. These exosomes may come from the MALAT1 overexpressed PBMCs, which can explain the increased exosomal miR-425 by sponging theory. But the plasma exosomes are a mixture, so the MALAT1 level in the pathological tissues needs to be examined.

In conclusion, this study identified two dysregulated noncoding RNAs (MALAT1 and miR-425) in patients with ARDS, and successfully constructed the linkage between upregulated cellular MALAT1, downregulated exosomal miR-425, and PTEN protein level in vitro. These findings exhibit a possible role of MALAT1 during the pathophysiological processes of ARDS which needs to be further confirmed in vivo.

\section{Data Availability}

The data used to support the findings of this study are available from the corresponding author upon request.

\section{Conflicts of Interest}

The authors declare that they have no conflicts of interest.

\section{Acknowledgments}

This work was supported by the Natural Science Foundation of Hubei Province of China (grant no. 2018CFB216) and the Fundamental Research Funds for the Central Universities (grant no. 2042018kf0174).

\section{Supplementary Materials}

Fig. S1: miRNA level in the plasma exosomes. Total RNAs were extracted from plasmal exosome and candidate miRNA level was detected by qRT-PCR. Results were shown as boxwhisker diagram, and data out of $95 \%$ CI are shown as dots. Results were analyzed by Student's $t$-test, and $p<0.05$ was considered significant. Fig. S2: miR-425 level was not reduced by MALAT1 in the cells. A549 and HFL-1 cells were transfected with wildtype or mutant MALAT1 expression vector for 48 hours. miR-425 level was detected by qRTPCR. (Supplementary Materials)

\section{References}

[1] R. E. Dechert, P. K. Park, and R. H. Bartlett, "Evaluation of the oxygenation index in adult respiratory failure," Journal of Trauma and Acute Care Surgery, vol. 76, no. 2, pp. 469-473, 2014.

[2] J. Villar, L. Perez-Mendez, S. Basaldua et al., "A risk tertiles model for predicting mortality in patients with acute respiratory distress syndrome: age, plateau pressure, and $\mathrm{PaO}_{2} /$ $\mathrm{FIO}_{2}$ at ARDS onset can predict mortality," Respiratory Care, vol. 56, no. 4, pp. 420-428, 2011.

[3] V. Galani, E. Tatsaki, M. Bai et al., "The role of apoptosis in the pathophysiology of Acute Respiratory Distress Syndrome (ARDS): an up-to-date cell-specific review," Pathology-Research and Practice, vol. 206, no. 3, pp. 145-150, 2010. 
[4] G. R. Bernard, A. Artigas, K. L. Brigham et al., "The AmericanEuropean consensus conference on ARDS. Definitions, mechanisms, relevant outcomes, and clinical trial coordination," American Journal of Respiratory and Critical Care Medicine, vol. 149, no. 3, pp. 818-824, 1994.

[5] V. M. Ranieri, G. D. Rubenfeld, B. T. Thompson et al., "Acute respiratory distress syndrome: the Berlin definition," JAMA, vol. 307, no. 23, pp. 2526-2533, 2012.

[6] L. B. Ware, J. A. Magarik, N. Wickersham et al., "Low plasma citrulline levels are associated with acute respiratory distress syndrome in patients with severe sepsis," Critical Care, vol. 17, no. 1, R10 pages, 2013.

[7] Y. Hu, J. Lin, H. Fang et al., "Targeting the MALAT1/PARP1/ LIG3 complex induces DNA damage and apoptosis in multiple myeloma," Leukemia, vol. 32, no. 10, pp. 2250-2262, 2018.

[8] S. Wu, H. Sun, Y. Wang et al., "MALAT1 Rs664589 polymorphism inhibits binding to miR-194-5p contributing to colorectal cancer risk, growth and metastasis," Cancer Research, 2019.

[9] X. Zhang, M. H. Hamblin, and K.-J. Yin, “The long noncoding RNA Malat1: its physiological and pathophysiological functions," RNA Biology, vol. 14, no. 12, pp. 1705-1714, 2017.

[10] X. Y. Ma, J. H. Wang, J. L. Wang, C. X. Ma, X. C. Wang, and F. S. Liu, "Malat1 as an evolutionarily conserved lncRNA, plays a positive role in regulating proliferation and maintaining undifferentiated status of early-stage hematopoietic cells," BMC Genomics, vol. 16, no. 1, 676 pages, 2015.

[11] L. Lei, Q. Zeng, J. Lu et al., "MALAT1 participates in ultraviolet $\mathrm{B}$-induced photo-aging via regulation of the ERK/ MAPK signaling pathway," Molecular Medicine Reports, vol. 15, no. 6, pp. 3977-3982, 2017.

[12] S. Sookoian, D. Flichman, M. E. Garaycoechea, J. San Martino, G. O. Castaño, and C. J. Pirola, "Metastasis-associated lung adenocarcinoma transcript 1 as a common molecular driver in the pathogenesis of nonalcoholic steatohepatitis and chronic immune-mediated liver damage," Hepatology Communications, vol. 2, no. 6, pp. 654-665, 2018.

[13] G. Zhao, Z. Su, D. Song, Y. Mao, and X. Mao, "The long noncoding RNA MALAT1 regulates the lipopolysaccharideinduced inflammatory response through its interaction with NF- $\kappa$ B," FEBS Letters, vol. 590, no. 17, pp. 2884-2895, 2016.

[14] L. Dai, G. Zhang, Z. Cheng et al., "Knockdown of LncRNA MALAT1 contributes to the suppression of inflammatory responses by up-regulating miR-146a in LPS-induced acute lung injury," Connective Tissue Research, vol. 59, no. 6, pp. 581-592, 2018.

[15] W. Meng, Y. Hao, C. He, L. Li, and G. Zhu, "Exosome-orchestrated hypoxic tumor microenvironment," Molecular Cancer, vol. 18, no. 1, 57 pages, 2019.

[16] A. V. Vlassov, S. Magdaleno, R. Setterquist, and R. Conrad, "Exosomes: current knowledge of their composition, biological functions, and diagnostic and therapeutic potentials," Biochimica et Biophysica Acta (BBA)_General Subjects, vol. 1820, no. 7, pp. 940-948, 2012.

[17] F. Yang, Z. Ning, L. Ma et al., "Exosomal miRNAs and miRNA dysregulation in cancer-associated fibroblasts," Molecular Cancer, vol. 16, no. 1, p. 148, 2017.

[18] X. Wu, Z. Liu, L. Hu, W. Gu, and L. Zhu, "Exosomes derived from endothelial progenitor cells ameliorate acute lung injury by transferring miR-126," Experimental Cell Research, vol. 370, no. 1, pp. 13-23, 2018.

[19] P. Zhang, H. Zhou, K. Lu, Y. Lu, Y. Wang, and T. Feng, "Exosome-mediated delivery of MALAT1 induces cell proliferation in breast cancer," OncoTargets and Therapy, vol. 11, pp. 291-299, 2018.

[20] N. A. Patel, L. D. Moss, J. Y. Lee et al., "Long noncoding RNA MALAT1 in exosomes drives regenerative function and modulates inflammation-linked networks following traumatic brain injury," Journal of Neuroinflammation, vol. 15, no. 1, 204 pages, 2018.

[21] K. M. Yamada and M. Araki, "Tumor suppressor PTEN: modulator of cell signaling, growth, migration and apoptosis," Journal of Cell Science, vol. 114, pp. 2375-2382, 2001.

[22] X. X. Lu, L. Y. Cao, X. Chen, J. Xiao, Y. Zou, and Q. Chen, "PTEN inhibits cell proliferation, promotes cell apoptosis, and induces cell cycle arrest via downregulating the PI3K/AKT/ hTERT pathway in lung adenocarcinoma A549 cells," BioMed Research International, vol. 2016, Article ID 2476842, 8 pages, 2016.

[23] A. Ittner, H. Block, C. A. Reichel et al., "Regulation of PTEN activity by $\mathrm{p} 38 \delta$-PKD1 signaling in neutrophils confers inflammatory responses in the lung," The Journal of Experimental Medicine, vol. 209, no. 12, pp. 2229-2246, 2012.

[24] Z. He, Y. Deng, W. Li et al., "Overexpression of PTEN suppresses lipopolysaccharide-induced lung fibroblast proliferation, differentiation and collagen secretion through inhibition of the PI3-K-Akt-GSK3beta pathway," Cell \& Bioscience, vol. 4, no. 1, 2 pages, 2014.

[25] M. N. Gong, B. T. Thompson, P. Williams, L. Pothier, P. D. Boyce, and D. C. Christiani, "Clinical predictors of and mortality in acute respiratory distress syndrome: potential role of red cell transfusion*," Critical Care Medicine, vol. 33, no. 6, pp. 1191-1198, 2005.

[26] Y. Li, Y. Xiang, Y. Song, L. Wan, G. Yu, and L. Tan, "Dysregulated miR-142, $-33 \mathrm{~b}$, and -423 in granulosa cells target TGFBR1 and SMAD7: a possible role in polycystic ovary syndrome," Molecular Human Reproduction, 2019.

[27] Y. Cao, Y. Lyu, J. Tang, and Y. Li, "MicroRNAs: novel regulatory molecules in acute lung injury/acute respiratory distress syndrome," Biomedical Reports, vol. 4, no. 5, pp. 523-527, 2016.

[28] C. Huang, X. Xiao, N. R. Chintagari, M. Breshears, Y. Wang, and L. Liu, "MicroRNA and mRNA expression profiling in rat acute respiratory distress syndrome," BMC Medical Genomics, vol. 7, no. 1, 46 pages, 2014.

[29] S. Rajasekaran, D. Pattarayan, P. Rajaguru, P. S. Sudhakar Gandhi, and R. K. Thimmulappa, "MicroRNA regulation of acute lung injury and acute respiratory distress syndrome," Journal of Cellular Physiology, vol. 231, no. 10, pp. 2097-2106, 2016.

[30] J. Barrera-Ramirez, J. R. Lavoie, H. B. Maganti et al., "MicroRNA profiling of exosomes from marrow-derived mesenchymal stromal cells in patients with acute myeloid leukemia: implications in leukemogenesis," Stem Cell Reviews and Reports, vol. 13, no. 6, pp. 817-825, 2017.

[31] J. Chen, C. Hu, and P. Pan, "Extracellular vesicle MicroRNA transfer in lung diseases," Frontiers in Physiology, vol. 8, p. 1028, 2017.

[32] H. YiRen, Y. YingCong, Y. Sunwu et al., "Long noncoding RNA MALAT1 regulates autophagy associated chemoresistance via miR-23b-3p sequestration in gastric cancer," Molecular Cancer, vol. 16, no. 1, 174 pages, 2017.

[33] W. Yan, Q. Wu, W. Yao et al., "MiR-503 modulates epithelialmesenchymal transition in silica-induced pulmonary fibrosis by targeting PI3K p85 and is sponged by lncRNA MALAT1," Scientific Reports, vol. 7, no. 1, Article ID 11313, 2017. 
[34] J. W. Christman, L. H. Lancaster, and T. S. Blackwell, "Nuclear factor $\mathrm{k}$ B: a pivotal role in the systemic inflammatory response syndrome and new target for therapy," Intensive Care Medicine, vol. 24, no. 11, pp. 1131-1138, 1998.

[35] Y. Ding, F. Guo, T. Zhu et al., "Mechanism of long non-coding RNA MALAT1 in lipopolysaccharide-induced acute kidney injury is mediated by the miR-146a/NF-kappaB signaling pathway," International Journal of Molecular Medicine, vol. 41, no. 1, pp. 446-454, 2018.

[36] V. Tripathi, J. D. Ellis, Z. Shen et al., "The nuclear-retained noncoding RNA MALAT1 regulates alternative splicing by modulating SR splicing factor phosphorylation," Molecular Cell, vol. 39, no. 6, pp. 925-938, 2010.

[37] R. Zhang, Y. Xia, Z. Wang et al., "Serum long non coding RNA MALAT-1 protected by exosomes is up-regulated and promotes cell proliferation and migration in non-small cell lung cancer," Biochemical and Biophysical Research Communications, vol. 490, no. 2, pp. 406-414, 2017.

[38] R. S. Patel, G. Carter, G. El Bassit et al., "Adipose-derived stem cells from lean and obese humans show depot specific differences in their stem cell markers, exosome contents and senescence: role of protein kinase C delta (PKCdelta) in adipose stem cell niche," Stem Cell Investigation, vol. 3, no. 2, 2016.

[39] J. Ma, J. Liu, Z. Wang et al., "NF-kappaB-dependent MicroRNA-425 upregulation promotes gastric cancer cell growth by targeting PTEN upon IL- $1 \beta$ induction," Molecular Cancer, vol. 13, no. 1, 40 pages, 2014

[40] F. Fang, T. Song, T. Zhang, Y. Cui, G. Zhang, and Q. Xiong, "MiR-425-5p promotes invasion and metastasis of hepatocellular carcinoma cells through SCAI-mediated dysregulation of multiple signaling pathways," Oncotarget, vol. 8, no. 19, pp. 31745-31757, 2017.

[41] U. Putz, J. Howitt, A. Doan et al., "The tumor suppressor PTEN is exported in exosomes and has phosphatase activity in recipient cells," Science Signaling, vol. 5, no. 243, ra70 pages, 2012.

[42] U. Putz, S. Mah, C.-P. Goh, L.-H. Low, J. Howitt, and S.-S. Tan, "PTEN secretion in exosomes," Methods, vol. 77-78, pp. 157-163, 2015. 


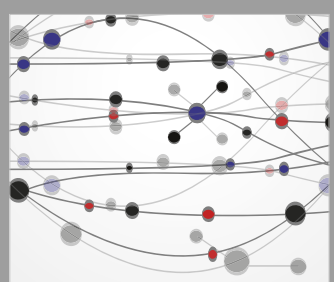

The Scientific World Journal
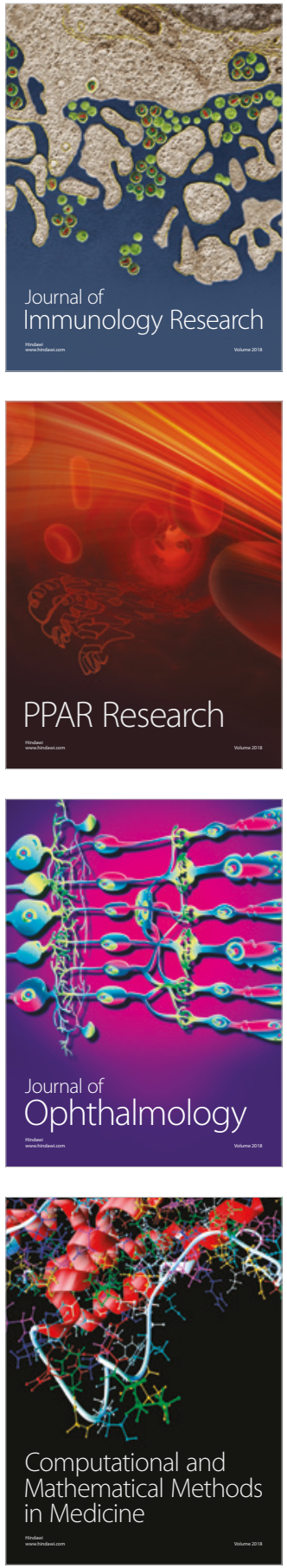

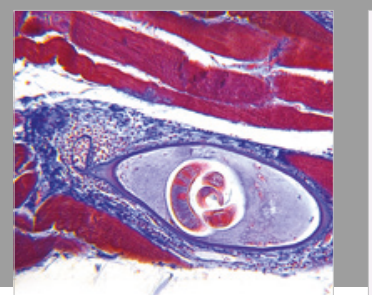

Gastroenterology Research and Practice

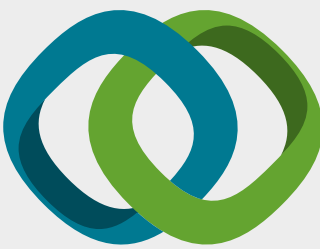

\section{Hindawi}

Submit your manuscripts at

www.hindawi.com
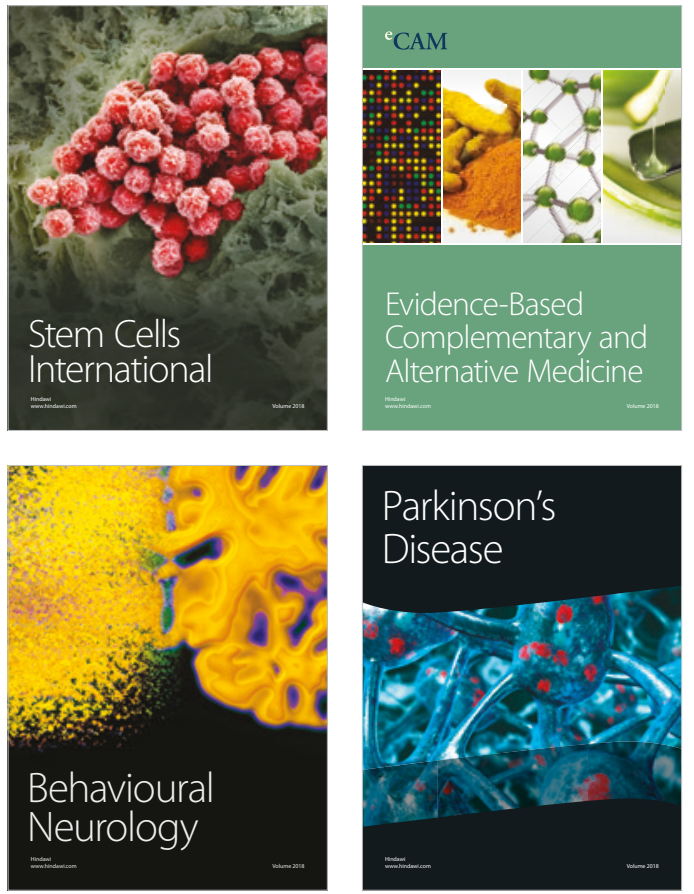

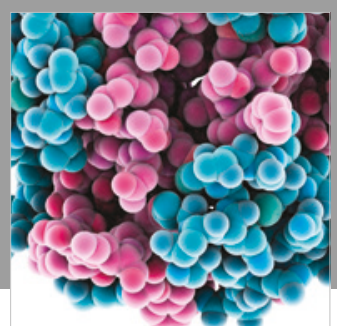

ournal of

Diabetes Research

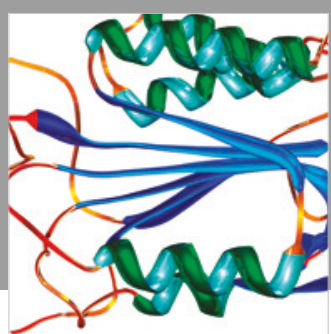

Disease Markers
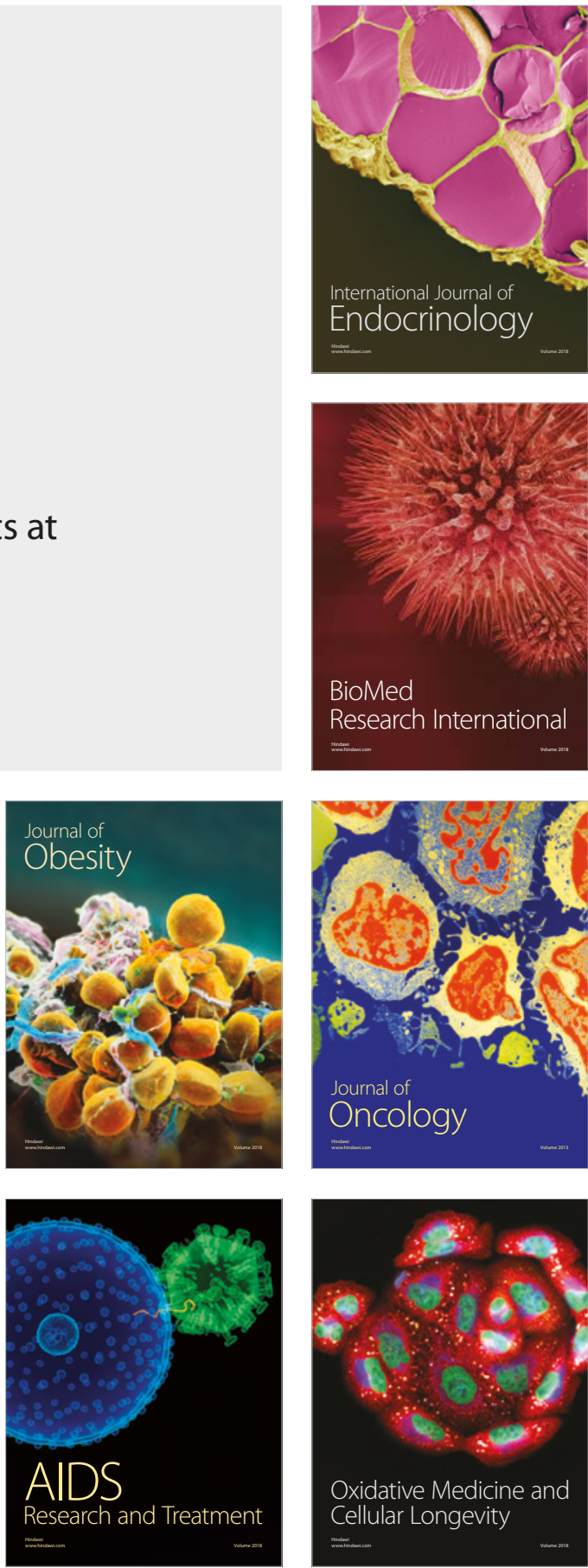